\title{
Novel Electromagnetic Phenomena in Graphene and Subsequent Microwave Devices Enabled by Multi-Scale Metamaterials
}

\author{
Dimitrios L. Sounas and Christophe Caloz \\ École Polytechnique de Montréal \\ Canada
}

\section{Introduction}

Metamaterials, i.e. artificial materials with properties not found in nature (Caloz \& Itoh, 2006; Marqués et al., 2008), have gained a lot of attention in the past decade. The virtually unlimited freedom in tailoring their constitutive parameters created hopes for the realization of unique devices, such as subwavelength lenses and invisibility cloaks, which were essentially impossible to achieve with classical materials. However, the implementation of such devices has been hindered by the inherent limitation of conventional metamaterials: non-perfect homogeneity due to the periodicity being insufficiently smaller than the wavelength, high insertion and thermal losses due to the conduction properties of the constituent elements and narrow bandwidth caused by the strongly resonant nature of the metamaterials constituent elements. Furthermore, the complex 3D structure of bulk metamaterials makes them unsuitable as microwave substrates and superstrates for planar devices where integration is a critical issue. Therefore, there is a need to develop a new generation of metamaterials with higher compactness and more homogeneity, exploiting the recent advances of nanotechnology, for instance in the fields of nanowires, nanopolymers, carbon nanotubes and graphene (Dragoman \& Dragoman, 2009; Hanson, 2007).

Graphene is a 2D material consisting of carbon atoms in a 2D honeycomb lattice (Geim \& Novoselov, 2007). It was first synthesized from graphite in the form of micro-flakes in 2004 (Novoselov et al., 2004) and since then it has been gaining a continuously increasing interest. Its gap-less linear band-structure results in unique phenomena, such as high electron mobility, ambipolarity, non-zero minimum conductivity and anomalous half-integer quantum Hall effect (Neto et al., 2009). Until now, a lot of research has been devoted to the design of electronic transport devices, such as transistors and non-linear components, mainly exploiting the high mobility and the ambipolar field effect of graphene (Lin et al., 2009; Wang et al., 2009). Recently, it has been demonstrated that graphene also possesses unique electromagnetic properties, such as low loss surface waves (Koppens et al., 2011; Mikhailov \& Ziegler, 2007; Vakil \& Engheta, 2011), huge Faraday rotation (Crassee et al., 2011; Sounas \& Caloz, 2011b) and super-confined edge magnetoplasmons (Mishchenko et al., 2010; Sounas \& Caloz, 2011a). These properties, combined with the ambipolar field effect which provides enhanced control capabilities of graphene's conductivity, open a new path towards super-compact and enhanced-functionality microwave devices. 
The chapter is organized as follows. Section 2 provides a brief overview of the electronic band structure of graphene, which is responsible for most of its unique properties. Section 3 introduces the surface conductivity model of graphene in the presence of a static magnetic field and gives a physical interpretation of the conductivity expressions. Section 4 constitutes the core of the chapter providing a theoretical analysis of Faraday rotation in graphene. In Section 5 two applications based on the gyrotropic properties of graphene, namely a circular waveguide Faraday rotator and a spatial isolator, are proposed. Section 6 describes a practical implementation of such devices through the multiscale metamaterials concept. Finally, Section 7 summarizes the chapter.

\section{Electronic band structure of graphene}

The unique properties of graphene originate from its electronic band structure. Using a tight-binding approximation and considering only the nearest-neighbor terms, the energy-wavevector dispersion relation of electrons in graphene is found as (Neto et al., 2009)

$$
E_{ \pm}\left(k_{x}, k_{y}\right)= \pm \sqrt{3+2 \cos \left(\sqrt{3} k_{y} a\right)+4 \cos \left(\frac{\sqrt{3}}{2} k_{y} a\right) \cos \left(\frac{3}{2} k_{x} a\right)}
$$

where $t=2.8 \mathrm{eV}$ is the nearest-neighbor hopping energy, $a=1.42 \AA$ is the interatomic distance, $k_{x}$ and $k_{y}$ are the $x$ and $y$ wavevector components, respectively, and the plus and minus signs refer to the upper (conduction) and the lower (valence) bands, respectively. Figs 1(a) and 1(b) plot $E$ versus $k_{x}$ and $k_{y}$ in the entire Brillouin zone and around the $K$ symmetry point, respectively. The $K$ and $K^{\prime}$ points are very important, since the energy at these points, which is 0 , is the chemical potential ${ }^{1}$ of intrinsic graphene. A zero chemical potential means a completely full valence band and a completely empty conduction band, hence minimal conductivity.

The first thing one can observe from Fig. 1 is the absence of an energy gap between the valence and the conduction bands. Because of this, graphene is usually referred to as a semi-metal ${ }^{2}$ or a zero-gap semiconductor ${ }^{3}$. The zero gap allows changing the type of conduction charge carriers from electrons to holes and vice versa by shifting the chemical potential from positive (conduction band) to negative (valence band) values, a phenomenon known as ambipolarity. The chemical potential can be easily tuned by an electrostatic voltage between graphene and an electrode parallel to it, as it is shown in Fig. 2. Assuming that the surface carrier density of graphene is $n_{s}$, the electric dispacement on both sizes of graphene is oriented perpendicularly to graphene, along the $z$ axis, and it has a value $D_{z}=\left(n_{s} e / 2\right) \operatorname{sgn}(z)$. The electric field in the substrate is then $E_{z}=D_{z} /\left(\varepsilon_{r} \varepsilon_{0}\right)=-n_{s} e /\left(2 \varepsilon_{r} \varepsilon_{0}\right)$. Integrating $E_{z}$ from graphene $(z=0)$ to the $\operatorname{PEC}(z=-d)$ plane, the apllied voltage is found as

$$
V_{g}=\frac{n_{s} e d}{2 \varepsilon_{r} \varepsilon_{0}} .
$$

\footnotetext{
${ }^{1}$ The chemical potential is the energy level with 0.5 probability of being fully occupied by electrons (the Fermi-Dirac distribution is 0.5). At zero temperature and under zero applied field (equilibrium), the chemical potential is equal to the Fermi level.

2 Metals have their conduction band penetrating into their valence band.

${ }^{3}$ Semiconductors have a non-zero gap between the valence and the conduction bands.
} 


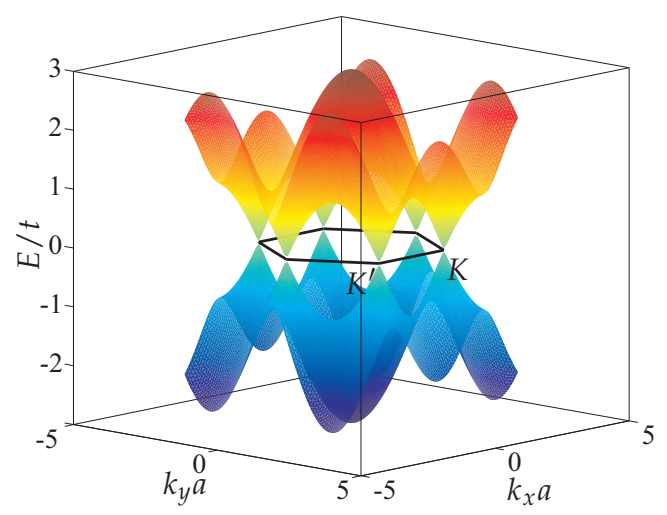

(a)

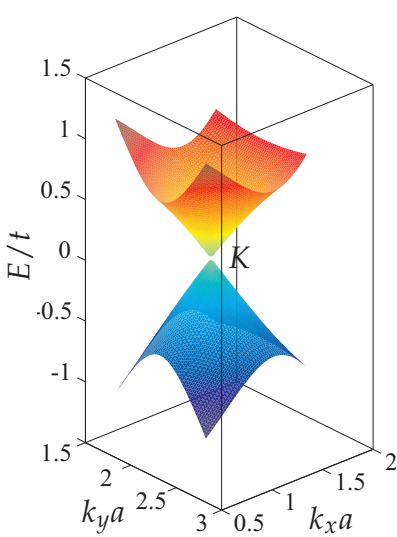

(b)

Fig. 1. Energy-wavenumber dispersion diagram of electrons in graphene. (a) Dispersion diagram in the entire Brillouin zone. (b) Dispersion diagram around the $K$ symmetry point (Dirac point).

Therefore, $n_{s}$, and subsequently $\mu_{c}$, can be controlled via $V_{g}$ (varying $V_{g}$ either provides electrons to graphene or depletes graphene from electrons). This way of controlling the carrier density is ofter called electrical doping, in contrast to chemical doping, where the carrier density is controlled by the density of impurity atoms introduced into the graphene lattice.

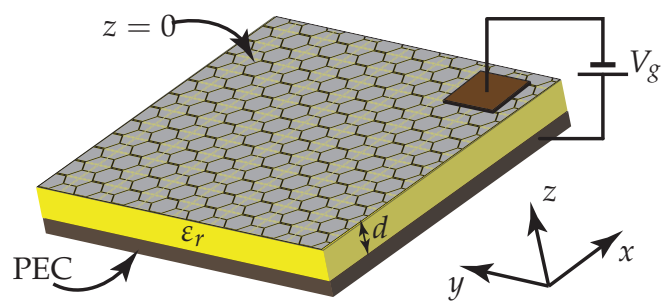

Fig. 2. Structure for controlling the chemical potential of graphene. A conducting electrode is placed at the bottom of graphene, forming with graphene a parallel plate-capacitor. An electrostatic voltage is applied between graphene and the electrode.

As already mentioned, the $K$ and $K^{\prime}$ points are of significant importance in graphene. Using a Taylor expansion around these points in Equation 1 and keeping only the first order term, the energy-wavevector dispersion relation takes the form

$$
E(\mathbf{q})= \pm \hbar v_{F}|\mathbf{q}|,
$$

where $\mathbf{q}=\mathbf{k}-\mathbf{k}_{\mathbf{K}, \mathbf{K}^{\prime}}$ and $v_{F}=3 t a /(2 \hbar) \approx 10^{6} \mathrm{~m} / \mathrm{s}$ is the Fermi velocity. Equation 3 is similar to the dispersion relation $E=\hbar c k$ of photons in vacuum and indicates that electrons in graphene behave like zero-mass particles (Dirac fermions) with an energy-independent velocity. As a result electrons in graphene exhibit quantum phenomena, such as the 
half-integer quantum Hall effect and the Klein paradox, normally only encountered in high energy relativistic particles.

Another important consequence of the graphene band structure, and more specifically of the double valley degeneracy associated with the $\mathrm{K}$ and $\mathrm{K}^{\prime}$ point, is the extremely low phonon scattering of electrons. This induces an upper limit of $200,000 \mathrm{~cm}^{2} / \mathrm{Vs}$ in the graphene mobility at room temperature and more than $1,000,000 \mathrm{~cm}^{2} / \mathrm{Vs}$ at low temperature! However, the practical mobility is much lower (it can be so low as $1,000 \mathrm{~cm}^{2} / \mathrm{Vs}$ ), due to various defects in the graphene lattice, such as impurities and wrinkles, and due to scattering from the substrate. Except for phonon scattering, which is related to the nature of the material and therefore imposes an upper bound on mobility, the scattering factors mainly depend on the production process. Therefore, improved production processes are expected to significantly increase the mobility to much higher values.

Biasing graphene with a static magnetic field $\mathbf{B}_{0}$ perpendicular to its plane results in quantization of the band diagram, as illustrated in Fig. 3. This phenomenon is called Landau quantization and it exists in all semiconductors. However, contrary to semiconductors with a parabolic band dispersion, the energy levels (Landau levels) in graphene are non-uniformly spaced, as a result of the linear dispersion close to the $K$ and $K^{\prime}$ points. Furthermore, the 0th order Landau level has a zero energy, irrespectively of the applied magnetic field, which is the reason for the observation of the quantum Hall effect in graphene even at room temperature. The energy of the $n$th Landau level in graphene is

$$
E_{n}=\sqrt{n} L
$$

where $L=\sqrt{2 \hbar e B_{0} v_{F}^{2}}$ is the Landau energy scale.

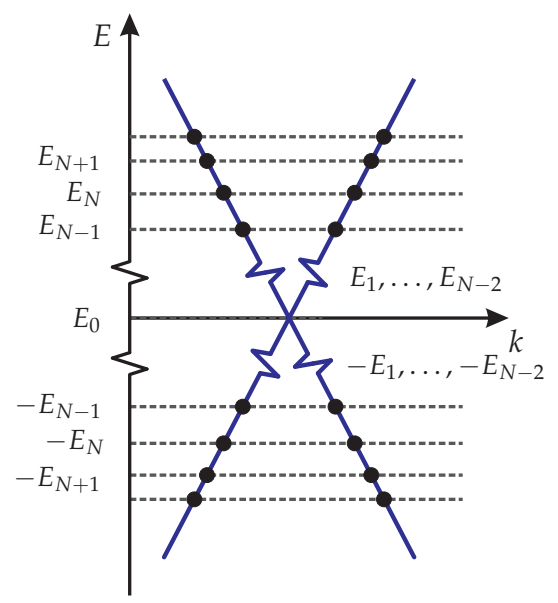

Fig. 3. Energy-wavenumber dispersion diagram in graphene, biased with a static magnetic field perpendicular to its plane. The dispersion diagram is quantized in the discrete energy levels $E_{0}, E_{ \pm 1}, \ldots$

Before closing this introductory section, let us provide the relation between the carrier density and the chemical potential, since the latter enters in all the conductivity expression that will 
be given in the next section. It is known from solid state physics that electrons are distributed in the energy levels of any material according to the Fermi-Dirac distribution

$$
f_{d}(E)=\frac{1}{1+e^{\left(E-\mu_{c}\right) /\left(k_{B} T\right)}},
$$

which represents the probability of finding an electron at the energy level $E$. In Equation $5 \mu_{c}$ is the chemical potential, $k_{B}$ is the Boltzmann constant and $T$ the temperature in K. Fig. 4 plots $f_{d}(E)$ for $\mu_{c}>0$, that is a partially filled conduction band. The electron and hole densities are then

$$
\begin{aligned}
& n_{s e}=\int_{0}^{\infty} f_{d}(E) N(E) d E \\
& n_{s h}=\int_{-\infty}^{0}\left[1-f_{d}(E)\right] N(E) d E,
\end{aligned}
$$

where

$$
N(E)=\frac{2 E}{\pi \hbar^{2} v_{F}^{2}}
$$

is the density of states. The density of states is the same for electrons and holes in graphene, due to the band-structure symmetry around the $E=0$ plane. The effective total carrier density is then $n_{s}=n_{s e}-n_{s h}$. Note that in intrinsic graphene $n_{s e}=n_{s h}$ and, therefore, $n_{s}=0$. Inserting Equation 5 into Equations 6 and then taking their difference yields

$$
n_{s}=\frac{2\left(k_{B} T\right)^{2}}{\pi \hbar^{2} v_{F}^{2}}\left[\operatorname{Li}_{2}\left(-e^{-\frac{\mu_{c}}{k_{B} T}}\right)-\operatorname{Li}_{2}\left(-e^{\frac{\mu_{c}}{k_{B} T}}\right)\right],
$$

where $\mathrm{Li}_{2}$ is the polylogarithm function of second order (Abramowitz \& Stegun, 1964). Inserting this relation into (2) provides the relation indicateing the required biasing voltage for a desired chemical potential. If $\mu_{c} \gg k_{B} T$, that is for highly doped graphene, Equation 8 takes the asymptotic form

$$
n_{s}=\frac{\mu_{c}^{2}}{\pi \hbar^{2} v_{F}^{2}}
$$

\section{Conductivity of magnetically biased graphene}

The essentially 2D structure of graphene makes surface conductivity the most natural appropriate quantity to model its electrical properties. When graphene is biased with a static magnetic field $\mathbf{B}_{0}$ perpendicular to its plane, conductivity takes a tensorial form, which may be deduced by considering the motion of an electron under an electric field $\mathbf{E}$ in the graphene plane, as illustrated in Fig. 5. For simplicity, the electron is considered to be initially at rest. Let us first examine the case $\mathbf{E}=E_{x} \hat{\mathbf{x}}$. The electric field exerts a force $\mathbf{F}_{e}=-e \hat{\mathbf{x}}$ on the electron, which accelerates it in the $-x$ direction. As long as the electron acquires a non-zero velocity, a magnetic force $\mathbf{F}_{m}=-e \mathbf{v} \times \mathbf{B}_{0}$ along the $-y$ direction is exerted on it and deflects it towards the $-y$ direction. The motion of the electron is, therefore, a combination of two simpler motions, one along the $-x$ axis and one along the $-y$ axis, and the electric current has two components, one along the $+x$ direction and one along the $+y$ direction ${ }^{4}$. For small

\footnotetext{
4 The current is opposite to the electron's velocity, due to the negative charge of the electron.
} 


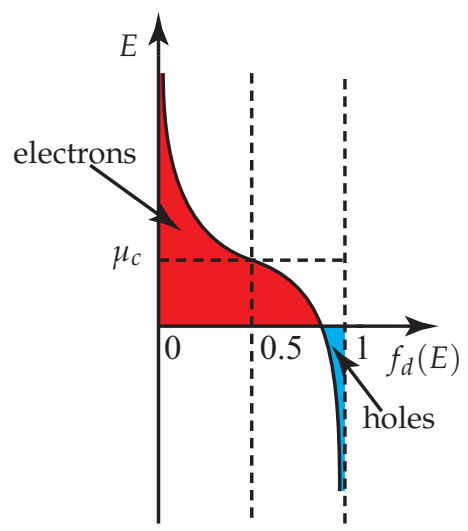

Fig. 4. Fermi-Dirac distribution for $\mu_{c}>0$. The red area corresponds to free electrons and the blue to free holes (electrons and holes that can conduct).

E, when nonlinear effects are negligible, the current can thus be related to the electric field through

$$
\mathbf{J}=\sigma_{x x} E_{x} \hat{\mathbf{x}}+\sigma_{y x} E_{x} \hat{\mathbf{y}} .
$$

The proportionality coefficients $\sigma_{x x}$ and $\sigma_{x y}$ correspond to the longitudinal (parallel to E) and transverse (perpendicular to E) conductivities, respectively, which depend on the band structure of the material, the frequency of $\mathbf{E}$ and $\mathbf{B}_{0}$. Following a similar analysis for $\mathbf{E}=E_{y} \hat{\mathbf{y}}$, which is the case depicted in Fig. 5(b), and assuming that graphene exhibits the same properties in all its directions, we derive

$$
\mathbf{J}=-\sigma_{y x} E_{y} \hat{\mathbf{x}}+\sigma_{x x} E_{x} \hat{\mathbf{y}} .
$$

Equations 10 and 11 can be combined into the single equation

$$
\mathbf{J}=\overline{\bar{\sigma}} \cdot \mathbf{E},
$$

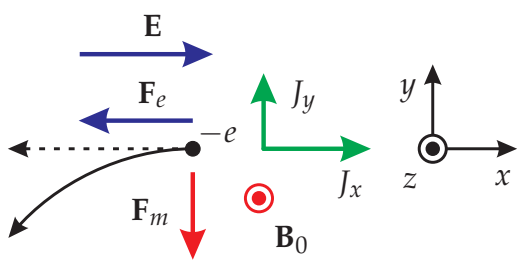

(a)

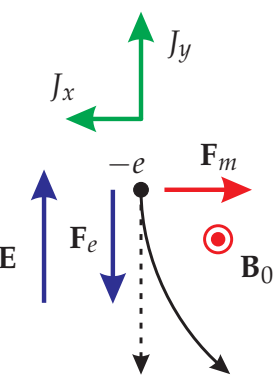

(b)

Fig. 5. Motion of an electron on graphene in an electric field and a static magnetic field. The electron, initially accelerated in the direction of the electric field, is deflected in the direction perpendicular to this field, due to the static magnetic field. Two electric current components, parallel and perpendicular to the electric field, are subsequently generated. (a) Case of the electric field along the $x$-axis. (b) Case of the electric field along the $y$-axis. 
where

$$
\overline{\bar{\sigma}}=\sigma_{x x} \overline{\bar{I}}_{t}+\sigma_{y x} \overline{\bar{J}}_{t}
$$

is the conductivity tensor of graphene and

$$
\begin{aligned}
& \overline{\bar{I}}_{t}=\hat{\mathbf{x}} \hat{\mathbf{x}}+\hat{\mathbf{y}} \hat{\mathbf{y}}, \\
& \overline{\bar{J}_{t}}=\hat{\mathbf{y}} \hat{\mathbf{x}}-\hat{\mathbf{x}} \hat{\mathbf{y}} .
\end{aligned}
$$

Closed-form expressions for $\sigma_{x x}$ and $\sigma_{y x}$ have been obtained in (Gusynin et al., 2009) through a quantum mechanical analysis which involves the Kubo formula (Kubo, 1957). Assuming an energy independent scattering mechanism, which is equivalent to considering an average scattering rate $\Gamma, \sigma_{x x}$ and $\sigma_{y x}$ are given by

$$
\begin{aligned}
\sigma_{x x}\left(\omega, B_{0}\right)= & \frac{e^{2} v_{F}^{2}\left|e B_{0}\right| \hbar(\omega-j 2 \Gamma)}{-j \pi} \sum_{n=0}^{\infty}\left\{\frac{1}{E_{n+1}-E_{n}}\right. \\
& \times \frac{f_{d}\left(E_{n}\right)-f_{d}\left(E_{n+1}\right)+f_{d}\left(-E_{n+1}\right)-f_{d}\left(-E_{n}\right)}{\left(E_{n+1}-E_{n}\right)^{2}-\hbar^{2}(\omega-j 2 \Gamma)^{2}} \\
& \left.+\left(E_{n} \rightarrow-E_{n}\right)\right\}, \\
\sigma_{y x}\left(\omega, B_{0}\right)= & -\frac{e^{2} v_{F}^{2} e B_{0}}{\pi} \sum_{n=0}^{\infty}\left[f_{d}\left(E_{n}\right)-f_{d}\left(E_{n+1}\right)-f_{d}\left(-E_{n+1}\right)+f_{d}\left(-E_{n}\right)\right] \\
& \times\left[\frac{1}{\left(E_{n+1}-E_{n}\right)^{2}-\hbar^{2}(\omega-j 2 \Gamma)^{2}}+\left(E_{n} \rightarrow-E_{n}\right)\right] .
\end{aligned}
$$

A physical explanation of Equation 15 may be provided with the help of Fig. 6. Each of the summation terms in Equation 15 corresponds to an electron transition between two Landau levels. Two types of transitions exist: intraband, between levels in the same band, and interband between levels of different bands. From all the possible intraband transitions, the only ones allowed by the selection rules are these from level $n$ to $n+1$ in the conduction band and from level $-n-1$ to $-n$ in the valence band. Similarly, the only allowed interband transitions are from level $-n$ to $n+1$ and from $-n-1$ to $n$. The photon energy needed for a transition between the levels $E_{\text {initial }}$ and $E_{\text {final }}$ is $E_{\text {final }}-E_{\text {initial }}$. A photon with this energy corresponds to an electromagnetic wave of frequency $\left(E_{\text {final }}-E_{\text {initial }}\right) / \hbar$. The probability of such a transition is proportional to the difference between the probability $f_{d}\left(E_{\text {initial }}\right)$ of the initial level being full and the probability $f_{d}\left(E_{\text {final }}\right)$ of the final level being empty. The transitions with the highest probabilities are those crossing $\mu_{c}$, across which the largest difference in $f_{d}(E)$ exists, emphasized by thicker arrows in Fig. 6. Therefore, assuming a finite temperature and that $\mu_{c}$ lies between the levels $E_{N}$ and $E_{N+1}$, the interband transitions with the lowest energy are the ones that involve the $-N$ and $N+1$ levels or the $-N-1$ and $N$ levels. As a result, interband transitions occur essentially at frequencies $\hbar \omega_{\text {inter }} \geq E_{N}+E_{N+1}$.

Assume now that $\mu_{c} \gg L$. For the magnetic field value of $1 \mathrm{~T}$, used in the chapter, $L=0.036 \mathrm{eV}$, and the condition $\mu_{c} \gg L$ is thus largely satisfied if $\mu_{c}>1 \mathrm{eV}$. Then $E_{N+1}>\mu_{c} \gg L$, which through Equation 4 yields $N \gg 1$ and $E_{N} \approx E_{N+1} \approx \mu_{c}$ (due to the $\sqrt{n}$ compression factor in $\left.E_{n}\right)$, resulting in $\hbar \omega_{\text {inter }} \geq 2 \mu_{c}$. In most of practical situations, $\mu_{c} \geq 0.05 \mathrm{eV}$, so that $\hbar \omega_{\text {inter }} \geq 0.15 \mathrm{PHz}$. Therefore, all the results of the chapter, which pertain to microwave and millimeter-wave operation, are derived by considering only the 


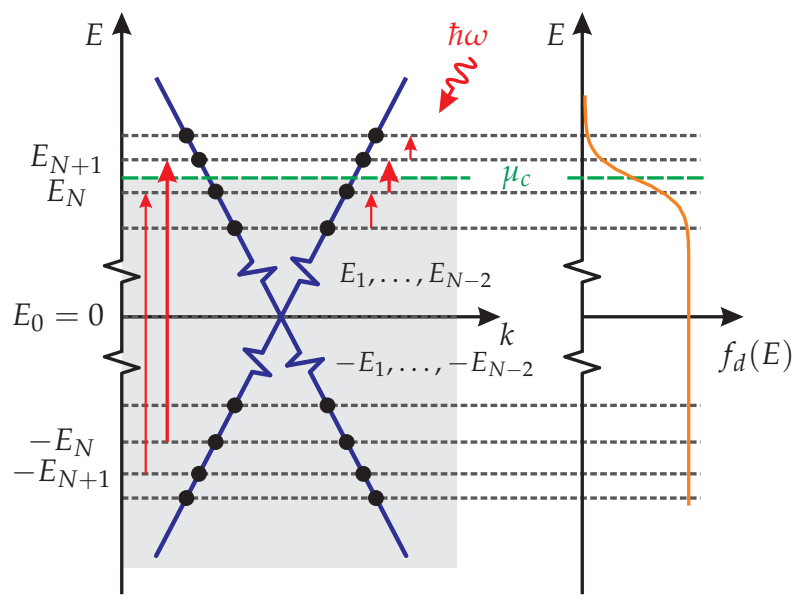

Fig. 6. Electron transitions in graphene when illuminated by an electromagnetic wave of frequency $\omega$. The red arrows represent the allowed intraband (right-hand side) and interband (left-hand side) electron transitions between the energy levels. A photon can be absorbed by graphene if its energy $\hbar \omega$ coincides with the energy of any of the allowed electron transitions. The probability of an electron transition depends on the probability of the initial level to be full and the probability of the final level to be empty. The probability of a level with energy $E$ to be full is given by the Fermi-Dirac distribution $f_{d}(E)$ (orange curve on the right).

intraband terms in Equation 15. Under the condition $N \gg 1$, the Landau levels around $\mu_{c}$, which produce non-negligible intraband transitions, lie very close to each other. It can be shown $(K u b o, 1957)$ that in this case $\sigma_{x x}$ and $\sigma_{y x}$ follow the Drude model form

$$
\begin{aligned}
& \sigma_{x x}\left(\omega, B_{0}\right)=\sigma_{0} \frac{1+j \omega \tau}{\left(\omega_{\mathcal{c}} \tau\right)^{2}+(1+j \omega \tau)^{2}}, \\
& \sigma_{y x}\left(\omega, B_{0}\right)=\sigma_{0} \frac{\omega_{\mathcal{c}} \tau}{\left(\omega_{\mathcal{c}} \tau\right)^{2}+(1+j \omega \tau)^{2}},
\end{aligned}
$$

where

$$
\sigma_{0}=\frac{2 e^{2} \tau}{\pi \hbar^{2}} k_{B} T \ln \left(2 \cosh \frac{\mu_{c}}{2 k_{B} T}\right)
$$

is the DC conductivity of graphene, $\tau=1 /(2 \Gamma)$ is the scattering time and $\omega_{C}$ is the cyclotron frequency. The latter corresponds to the difference between the levels $E_{N}$ and $E_{N+1}$,

$$
\omega_{c}=\frac{E_{N+1}-E_{N}}{\hbar}=\frac{L}{\hbar(\sqrt{N+1}+\sqrt{N})} \approx \frac{L^{2}}{2 \hbar \mu_{c}}=\frac{e B_{0} v_{F}^{2}}{\mu_{c}} .
$$

For the $B_{0}$ values used in the chapter, $\mu_{c} \gg L$ also implies $\mu_{c} \gg k_{B} T\left(k_{B} T=0.026 \mathrm{eV}\right.$ at $\left.300 \mathrm{~K}\right)$, and Equation 17 subsequently simplifies to $\sigma_{0}=n_{s} e \mu$, where $n_{s}=\mu_{c}^{2} / \pi \hbar^{2} v_{F}^{2}$ is the carrier density and $\mu=e \tau v_{F}^{2} / \mu_{c}$ the mobility. Figure 7 plots $\sigma_{x x}$ and $\sigma_{y x}$ versus frequency using both Equation 15 and 16, for $B_{0}=1 \mathrm{~T}, \mu=5000 \mathrm{~cm}^{2} / \mathrm{Vs}, n_{\mathrm{s}}=10^{13} \mathrm{~cm}^{-2}$ and $T=300 \mathrm{~K}$, corresponding to values widely used throughout the chapter. Excellent agreement between the two models is observed in the frequency range shown. 


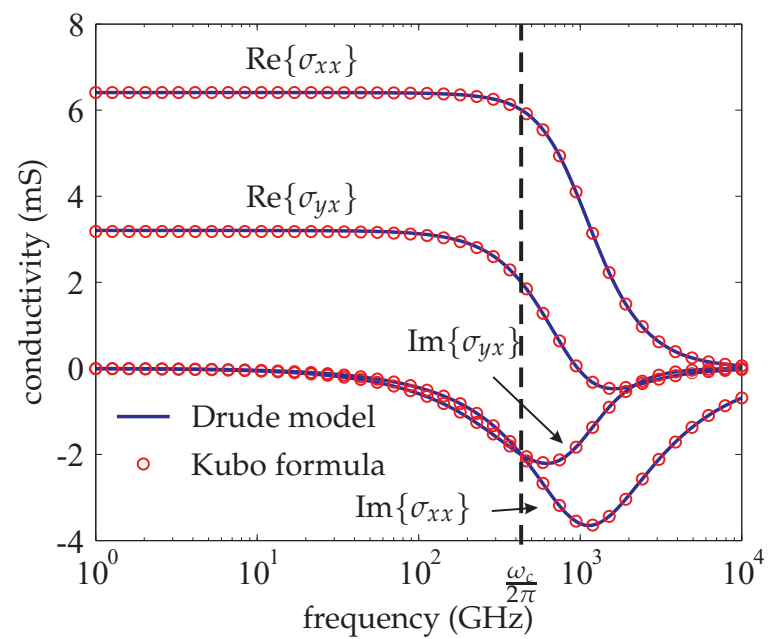

Fig. 7. Graphene conductivity versus frequency computed from the exact expressions 15 (circles) and from the approximate expressions 16 (lines), for $\mu=5000 \mathrm{~cm}^{2} / \mathrm{Vs}$, $n_{\mathrm{s}}=10^{13} \mathrm{~cm}^{-2}, B_{0}=1 \mathrm{~T}$ and $T=300 \mathrm{~K}$.

\section{Faraday rotation in graphene}

When a plasma, such as a semiconductor, is biased with a static magnetic field it exhibits Faraday rotation at frequencies above the cyclotron frequency. The reason why Faraday rotation is only observed above the cyclotron resonance is that below the resonance the plasma is impenetrable. Graphene, being a semiconductor, is also expected to exhibit Faraday rotation (Crassee et al., 2011; Sounas \& Caloz, 2011b). However, contrary to semiconductors, Faraday rotation in graphene exists also below the cyclotron resonance, as a result of its super-thin, semi-transparent nature. This section theoretically analyzes Faraday rotation in graphene. The results will be used in the next section where possible applications of this phenomenon will be suggested.

Consider a graphene sheet coinciding with the $z=0$ plane in free space and biased with a static magnetic field $\mathbf{B}_{0}=B_{0} \hat{\mathbf{z}}$ and a plane wave impinging normally on the sheet from medium 1 towards medium 2, as illustrated in Fig. 8. The electric field of the incident, reflected and transmitted waves then read

$$
\begin{aligned}
\mathbf{E}^{\text {inc }} & =\mathbf{E}_{0}^{\text {inc }} e^{-j k_{0} z}, \\
\mathbf{E}^{\text {ref }} & =\mathbf{E}_{0}^{\text {ref }} e^{j k_{0} z}, \\
\mathbf{E}^{\text {tran }} & =\mathbf{E}_{0}^{\text {tran }} e^{-j k_{0} z} .
\end{aligned}
$$

In order to find $\mathbf{E}_{0}^{\text {ref }}$ and $\mathbf{E}_{0}^{\text {tran }}$, we will use the boundary conditions

$$
\begin{aligned}
\hat{\mathbf{z}} \times\left.\left(\mathbf{E}^{\operatorname{tran}}-\mathbf{E}^{\mathrm{inc}}-\mathbf{E}^{\mathrm{ref}}\right)\right|_{z=0}=\mathbf{0}, \\
\hat{\mathbf{z}} \times\left.\left(\mathbf{H}^{\text {tran }}-\mathbf{H}^{\mathrm{inc}}-\mathbf{H}^{\mathrm{ref}}\right)\right|_{z=0}=\left.\overline{\bar{\sigma}} \cdot \mathbf{E}^{\operatorname{tran}}\right|_{z=0^{\prime}}
\end{aligned}
$$


which hold in the graphene plane. The magnetic fields $\mathbf{H}^{\text {inc }}, \mathbf{H}^{\text {ref }}$ and $\mathbf{H}^{\text {tran }}$ are related to the corresponding electric fields through

$$
\begin{aligned}
\mathbf{H}^{\text {inc }} & =\frac{\hat{\mathbf{z}} \times \mathbf{E}^{\text {inc }}}{\eta_{0}}, \\
\mathbf{H}^{\text {ref }} & =-\frac{\hat{\mathbf{z}} \times \mathbf{E}^{\text {inc }}}{\eta_{0}}, \\
\mathbf{H}^{\text {tran }} & =\frac{\hat{\mathbf{z}} \times \mathbf{E}^{\text {inc }}}{\eta_{0}},
\end{aligned}
$$

where $\eta_{0}$ is the free-space wave impedance. Inserting Equation 19 into 20a yields

$$
\mathbf{E}_{0}^{\text {tran }}-\mathbf{E}_{0}^{\text {inc }}-\mathbf{E}_{0}^{\text {tran }}=\mathbf{0} .
$$

Similarly, inserting Equation 21 into 20b, one gets

$$
-\mathbf{E}_{0}^{\text {tran }}+\mathbf{E}_{0}^{\text {inc }}-\mathbf{E}_{0}^{\text {ref }}=\eta_{0} \overline{\bar{\sigma}} \cdot \mathbf{E}_{0}^{\text {tran }} .
$$

Solving Equations 22 and $20 \mathrm{~b}$ simultaneously with respect to $\mathbf{E}_{0}^{\text {tran }}$ gives

$$
\mathbf{E}_{0}^{\operatorname{tran}}=2\left(2 \overline{\bar{I}}_{t}+\eta_{0} \overline{\bar{\sigma}}\right)^{-1} \cdot \mathbf{E}_{0}^{\text {inc }} .
$$

Therefore, the transmission dyadic for incidence from medium 1 towards medium 2 is

$$
\overline{\bar{T}}_{21}=2\left(2 \overline{\bar{I}}_{t}+\eta_{0} \overline{\bar{\sigma}}\right)^{-1}=\frac{2}{\left(2+\eta_{0} \sigma_{x x}\right)^{2}+\left(\eta_{0} \sigma_{y x}\right)^{2}}\left[\left(2+\eta_{0} \sigma_{x x}\right) \overline{\bar{I}}_{t}-\eta_{0} \sigma_{y x} \overline{\bar{J}}_{t}\right],
$$

where the dyadic identity (Lindell, 1996)

$$
\left(a \overline{\bar{I}}_{t}+b \overline{\bar{J}}_{t}\right)^{-1}=\frac{1}{a^{2}+b^{2}}\left(a \overline{\bar{I}}_{t}-b \overline{\bar{J}}_{t}\right)
$$

has been used. Through a similar analysis, it can be shown that the transmission dyadic for incidence from medium 2 towards medium 1 is

$$
\overline{\bar{T}}_{12}=\overline{\bar{T}}_{21}
$$

a condition which, as it will be shown later, indicates non-reciprocity.

Consider now the specific case of a left-handed (LH) circularly polarized incident wave with respect to the $z$-axis $\mathrm{E}_{\mathrm{LH}, 0}^{\mathrm{inc}}=\hat{\mathbf{x}}+j \hat{\mathbf{y}}$. The transmitted wave is then, according to Equation 25,

$$
\mathbf{E}_{\mathrm{LH}, 0}^{\operatorname{tran}}=\overline{\bar{T}}_{21} \cdot \mathbf{E}_{\mathrm{LH}, 0}^{\mathrm{inc}}=\frac{2}{2+\eta_{0} \sigma_{\mathrm{LH}}} \mathbf{E}_{\mathrm{LH}, 0}^{\mathrm{inc}}
$$

where $\sigma_{\mathrm{LH}}=\sigma_{x x}-j \sigma_{y x}$. Therefore, in the case of a LH circularly polarized wave the transmission coefficient simplifies to

$$
T_{\mathrm{LH}}=\frac{2}{2+\eta_{0} \sigma_{\mathrm{LH}}} .
$$




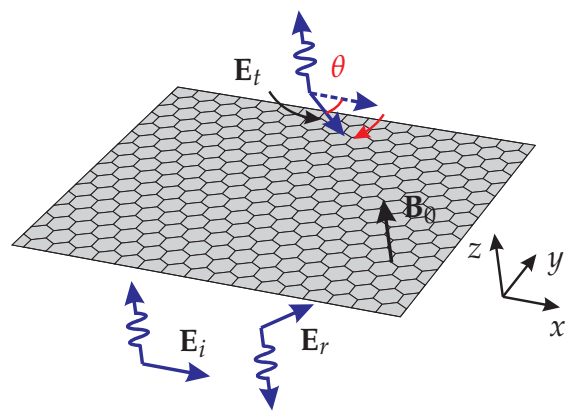

Fig. 8. Magnetically biased graphene sheet in the $z=0$ plane. A plane wave impinges normally on graphene.

Similarly, the transmission coefficient for a right-handed $(\mathrm{RH})$ circularly polarized wave is

$$
T_{\mathrm{RH}}=\frac{2}{2+\eta_{0} \sigma_{\mathrm{RH}}},
$$

where $\sigma_{R H}=\sigma_{x x}+j \sigma_{y x}$. Equations 29 and 30 reveal that $T_{\mathrm{LH}} \neq T_{\mathrm{RH}}$, a phenomenon known as circular birefringence, with Faraday rotation being one of its most important consequences.

Fig. 9 plots the amplitude and phase of $T_{\mathrm{LH}}$ and $T_{\mathrm{RH}}$ versus frequency for a graphene sheet with $\tau=1.84 \times 10^{-13} \mathrm{~s}, \mu_{c}=0.37 \mathrm{eV}$ and $B_{0}=1 \mathrm{~T}$. Two things can be observed: a) the amplitudes of $T_{\mathrm{LH}}$ and $T_{\mathrm{RH}}$ are almost the same far from the cyclotron resonance and $b$ ) there is a huge phase difference between $T_{\mathrm{LH}}$ and $T_{\mathrm{RH}}$ below the cyclotron resonance. These two facts result in a large Faraday rotation below the cyclotron resonance. To prove this statement, consider an $x$-polarized incident wave $\mathbf{E}_{0}^{\text {inc }}=\hat{\mathbf{x}}$. Such a wave can be decomposed into a $\mathrm{RH}$ circularly polarized wave and a LH circularly polarized wave as

$$
\mathbf{E}^{\text {inc }}=\frac{1}{2}(\hat{\mathbf{x}}+j \hat{\mathbf{y}})+\frac{1}{2}(\hat{\mathbf{x}}-j \hat{\mathbf{y}}) .
$$

The transmitted wave is then

$$
\mathbf{E}^{\operatorname{tran}}=\frac{T_{\mathrm{LH}}}{2}(\hat{\mathbf{x}}+j \hat{\mathbf{y}})+\frac{T_{\mathrm{RH}}}{2}(\hat{\mathbf{x}}-j \hat{\mathbf{y}}) .
$$

If $\left|T_{\mathrm{LH}}\right| \approx\left|T_{\mathrm{RH}}\right|$, as in the case far below and above from the cyclotron resonance, Equation 32 becomes

$$
\begin{aligned}
\mathbf{E}^{\operatorname{tran}} & =\frac{\left|T_{\mathrm{LH}}\right|}{2}\left[e^{j \varphi_{\mathrm{LH}}}(\hat{\mathbf{x}}+j \hat{\mathbf{y}})+e^{j \varphi_{\mathrm{RH}}}(\hat{\mathbf{x}}-j \hat{\mathbf{y}})\right] \\
& =\frac{\left|T_{\mathrm{LH}}\right|}{2} e^{j \varphi_{\mathrm{av}}}\left[\hat{\mathbf{x}} \cos \left(\frac{\Delta \varphi}{2}\right)-\hat{\mathbf{y}} \sin \left(\frac{\Delta \varphi}{2}\right)\right],
\end{aligned}
$$

where $\varphi_{\mathrm{LH}}=\arg \left\{T_{\mathrm{LH}}\right\}, \varphi_{\mathrm{RH}}=\arg \left\{T_{\mathrm{RH}}\right\}, \varphi_{\mathrm{av}}=\left(\varphi_{\mathrm{LH}}+\varphi_{\mathrm{RH}}\right) / 2$ and $\Delta \varphi=\varphi_{\mathrm{LH}}-\varphi_{\mathrm{RH}}$. Since $\Delta \varphi>0$ (Fig. 9) the field has been rotated by an angle

$$
\theta=\frac{\Delta \varphi}{2}
$$


in the $\mathrm{RH}$ direction with respect to the $+z$-direction (the direction of the magnetic field). For $\omega \ll \omega_{c}$, where $\sigma_{x x}$ and $\sigma_{y x}$ are almost purely real, the phases of $T_{\mathrm{LH}}$ and $T_{\mathrm{RH}}$ are found from Equations 29 and 30 as

$$
\begin{aligned}
\varphi_{\mathrm{LH}} & =\tan ^{-1}\left(\frac{\eta_{0} \sigma_{y x}}{2+\eta_{0} \sigma_{x x}}\right) \\
\varphi_{\mathrm{RH}} & =-\tan ^{-1}\left(\frac{\eta_{0} \sigma_{y x}}{2+\eta_{0} \sigma_{x x}}\right) .
\end{aligned}
$$

Thus, for $\omega \ll \omega_{c}$, the rotation angle is

$$
\theta=\tan ^{-1}\left(\frac{\eta_{0} \sigma_{y x}}{2+\eta_{0} \sigma_{x x}}\right)
$$

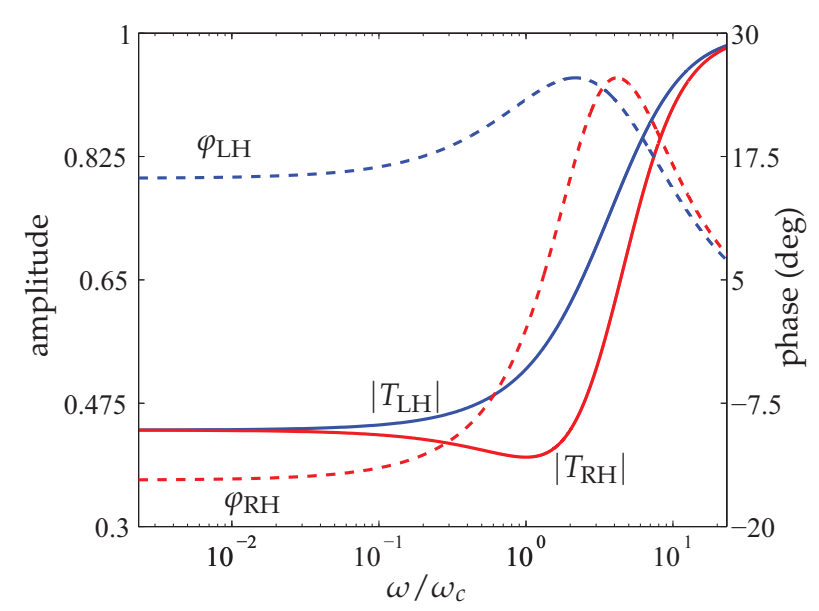

Fig. 9. Transmission coefficient for normal incidence of a $\mathrm{LH}$ and a $\mathrm{RH}$ circularly polarized wave on a graphene sheet in free space with $\tau=1.84 \times 10^{-13} \mathrm{~s}, \mu_{c}=0.37 \mathrm{eV}$ and $B_{0}=1 \mathrm{~T}$.

Fig. 10 presents $\theta$ and the transmission amplitude $\left(\left|T_{\mathrm{LH}}\right| \approx\left|T_{\mathrm{RH}}\right|\right)$ versus $\mu_{c}$ and $B_{0}$ for a graphene sheet with $\tau=1.84 \times 10^{-13} \mathrm{~s}$ at $30 \mathrm{GHz}$ and $T=300 \mathrm{~K}$. The rotation angle clearly depends on both $\mu_{c}$ and $B_{0}$, providing two degrees of freedom in controlling it. Regarding the $\mu_{c}$ dependence, $\theta \rightarrow 0$ as $\mu_{c} \rightarrow 0$. This is expected from the fact that for $\mu_{c}=0$ there are very few electrons in the conduction band, hence the conductivity is very small and the interaction between the electromagnetic waves and the material is negligible. As $\mu_{c}$ increases, $\theta$ also increases up to a specific $\mu_{c}$ value, which depends on $B_{0}$. As $\mu_{c}$ further increases, $\theta$ decreases and tends to 0 for very large $\mu_{c}$, because the conductivity becomes very large and the material behaves like a perfect electric conductor. Concerning the $B_{0}$ dependence, Fig. 10 indicates that $\theta$ increases along a line of constant transmission amplitude. For $\mu_{c}$ fixed, $\theta$ increases linearly with $B_{0}$ when $\omega_{\mathcal{c}} \tau \ll 1$, it becomes maximum when $\omega_{\mathcal{c}} \tau \approx 1$ and it decreases inversely proportionally with $B_{0}$ when $\omega_{c} \tau \gg 1$.

In addition to controlling the amount of rotation, we can also control its direction via $\mu_{c}$, exploiting the ambipolar properties of graphene. Specifically, by inverting $\mu_{c}$ from positive to negative values, the type of charge carriers changes from electrons to holes. Holes have 


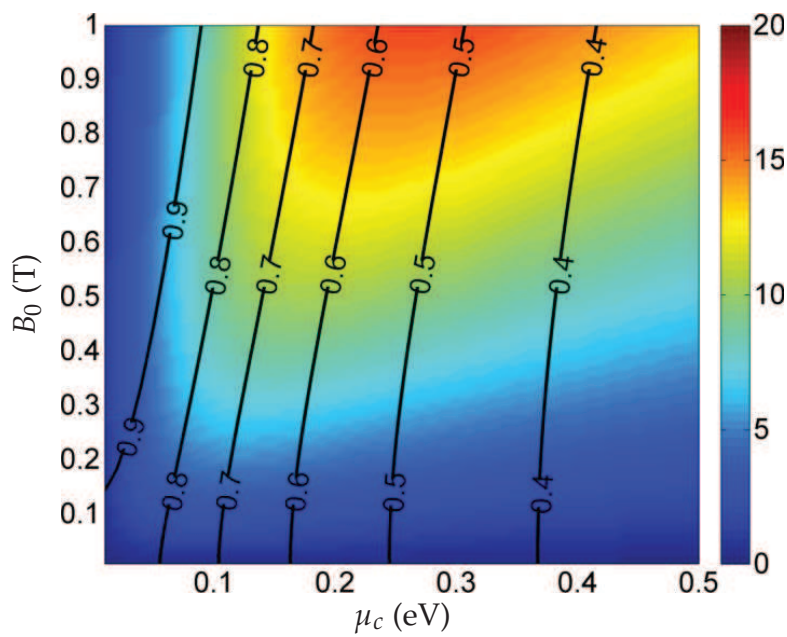

Fig. 10. Rotation angle (color plot) and transmission amplitude (contour plot) versus $\mu_{c}$ and $B_{0}$ for a normally incident plane wave on a graphene sheet in free space, for the parameters $\tau=1.84 \times 10^{-13} \mathrm{~s}, f=30 \mathrm{GHz}$, and $\mathrm{T}=300 \mathrm{~K}$.

an opposite charge than electrons and, therefore, provide an opposite transverse current than electrons, hence an opposite rotation direction, as illustrated in Fig. 11. This property is of significant practical importance, since it allows the control of the rotation direction via an electrostatic potential applied to graphene, as shown in Fig. 2, while keeping the magnetic field constant. Note that the static magnetic field is usually provided by permanent magnets and therefore it is extremely difficult to invert.

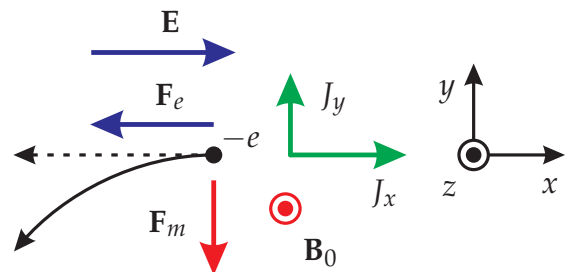

(a)

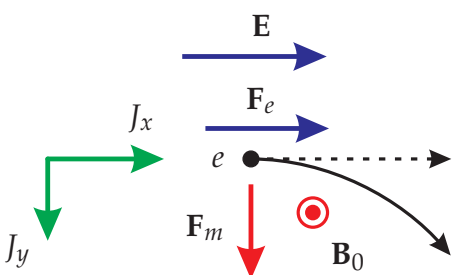

(b)

Fig. 11. Reversal of the direction of cyclotron rotation resulting from reversal of the type of charge carriers. (a) Case of electrons carriers. (b) Case of hole carriers, where the the transverse current has been reversed due to the reversal of the charge sign.

Although neglected until now, the fact that $\sigma_{x x}$ and $\sigma_{y x}$ are not purely real or purely imaginary numbers results in the transmitted wave being elliptically instead of linearly polarized. Fig. 12 plots $\theta$ and the axial ratio of the transmitted wave versus frequency for $\tau=1.84 \times 10^{-13} \mathrm{~s}$, $\mu_{c}=0.37 \mathrm{eV}, B_{0}=1 \mathrm{~T}$ and $T=300 \mathrm{~K}$. For $\omega \ll \omega_{c}$, when $\sigma_{x x}$ and $\sigma_{y x}$ are almost purely real, the axial ratio is very high, indicating a linearly polarized wave. As frequency increases, the axial ratio decreases and it becomes minimum close to the cyclotron resonance, where 


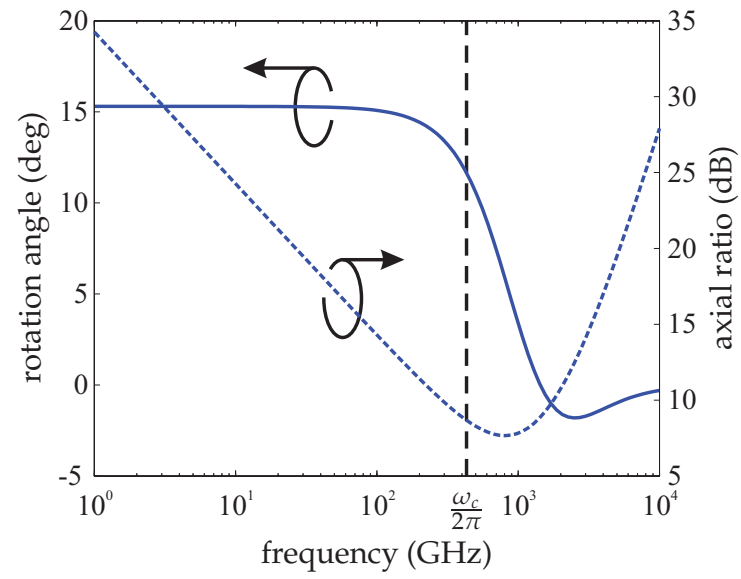

Fig. 12. Rotation angle, computed by Equation 34, and axial ratio, computed from Equation 25 and Balanis (2005), versus frequency for a normally incident plane wave on a graphene sheet in free space, for the parameters $\tau=1.84 \times 10^{-13} \mathrm{~s}, \mu_{c}=0.37 \mathrm{eV}, B_{0}=1 \mathrm{~T}$, and $T=300 \mathrm{~K}$.

there is a strong interaction between the material and the circular polarization of the same handedness as the cyclotron motion of electrons. For a further increase of frequency this "resonance" between the circularly polarized waves and the electrons diminishes gradually and the axial ratio increases.

Fig. 12 also shows that Faraday rotation in graphene is an extremely broadband phenomenon below the resonance (in the case of Fig. 12 from 0 to $200 \mathrm{GHz}$ ), as a result of the non-dispersive characteristics of conductivity below the resonance (Fig. 7). It has to be stressed that this frequency-independent behavior is different from the corresponding found in ferrites above the ferromagnetic resonance (Lax \& Button, 1962), since the former is due to the non-dispersive characteristics of the material while the latter is due to the increase of the electrical length with frequency.

The direction of Faraday rotation in graphene, as in any magneto-optical material, does not depend on the propagation direction but on the direction of the applied static magnetic field. This can be directly seen from $\overline{\bar{T}}_{21}=\overline{\bar{T}}_{12}$ (Equation 27), which states that for an incident wave of a given polarization the polarization of the transmitted wave is always the same, irrespectively of the propagation direction. Thus, graphene is a non-reciprocal material. In order to demonstrate this statement, consider a plane wave impinging on graphene from medium 1 to medium 2 and then reflected back to medium 1 without changing its polarization (e.g. reflection by a PMC wall after graphene), as illustrated in Fig. 13. As the wave passes through graphene from medium 1 towards medium 2, its polarization is rotated by an angle $\theta$ in the LH direction with respect to the $+z$ direction (the biasing magnetic field direction). As the wave then passes again through graphene from medium 2 back to medium 1, its polarization is again rotated by an angle $\theta$ in the LH direction with respect to the $+z$ axis. Therefore, the polarization of the wave transmitted from medium 1 to medium 2 and then back from medium 2 to medium 1 has undergone an overall rotation of $2 \theta$, which is a manifestation of non-reciprocity. 


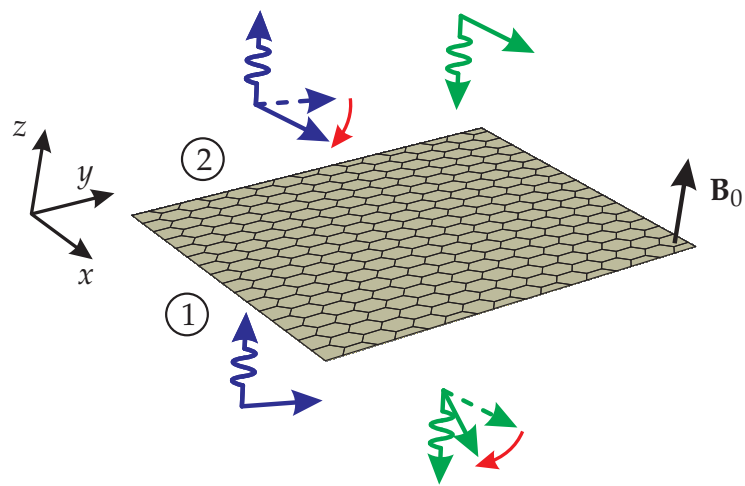

Fig. 13. Non-reciprocity in graphene. A wave normally impinging on graphene from medium 1 to medium 2 and transmitted back to medium 1 undergoes a total rotation angle of $2 \theta$, where $\theta$ is the rotation angle for a single pass through graphene.

\section{Applications}

In this section, two applications of the Faraday rotation effect in graphene will be presented, namely a circular waveguide polarization rotator and a non-reciprocal spatial isolator. Although such devices can be realized with conventional gyrotropic media, such as ferrites, graphene offers increased tunability and electric rotation reversal, and possibly other benefits (such as high heat sink) still to be investigated. The purpose of the section is to show the feasibility of using graphene in such types of devices and not to provide an optimized design. Therefore, the dimensions of the proposed devices are comparable to their ferrite counterparts. However, we believe that by a proper design, e.g. by using a stacked structure composed of alternating graphene sheets and dielectric layers, highly compact devices, with dimensions much smaller than the dimensions of the ferrite devices, can be achieved.

\subsection{Circular waveguide faraday rotator}

The proposed circular waveguide Faraday rotator consists of a graphene sheet loading the cross section of a circular waveguide, as shown in Fig. 14. The graphene sheet is perpendicular to the waveguide axis and biased with an axial static magnetic field $\mathbf{B}_{0}$. The waveguide has a radius $a$ and it is excited in its dominant $\mathrm{H}_{11}\left(\mathrm{TE}_{11}\right)$ mode. The incident mode is assumed to be polarized along the $x$ axis and propagates along the $+z$ direction.

The transverse electric field of the mode is

$$
\mathbf{E}_{T, 11}^{\mathrm{h}, \text { inc }}(\rho, \varphi)=\hat{\mathbf{z}} \times \nabla_{t} \times \psi_{11}(\rho, \varphi),
$$

where $\nabla_{t}$ is the transverse (on the $\rho-\varphi$ plane) del operator and $\psi_{11}(\rho, \varphi)=J_{1}\left(k_{c, 11}^{h} \rho\right) \cos \varphi$, with $k_{c, 11}^{h}=x_{11}^{\prime} / a$ and $x_{11}^{\prime}$ the first root of $J_{1}^{\prime}(x)$. Using $\cos \varphi=\left(e^{j \varphi}+e^{-j \varphi}\right) / 2$, the incident mode can be analyzed into two degenerate orthogonal modes, a LH circularly polarized mode $\mathrm{H}_{11}^{-}$

$$
\mathbf{E}_{T, 11}^{h-}=\hat{\mathbf{z}} \times \nabla_{t} \times \psi_{11}^{-}
$$

and a $\mathrm{RH}$ circularly polarized one $\mathrm{H}_{11}^{+}$

$$
\mathbf{E}_{T, 11}^{h+}=\hat{\mathbf{z}} \times \nabla_{t} \times \psi_{11}^{+},
$$




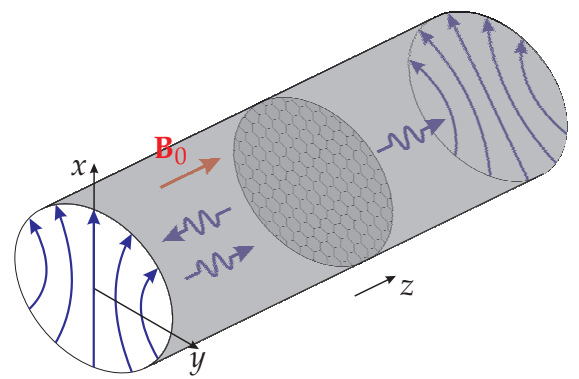

Fig. 14. Circular cylindrical waveguide of radius $a$ loaded by a graphene sheet perpendicular to its axis. The sheet is placed at the $z=0$ plane and biased by a perpendicular static magnetic field $B_{0}$. The waveguide is excited in its dominant $\mathrm{H}_{11}\left(\mathrm{TE}_{11}\right)$ mode.

both with amplitude equal to $1 / 2$. In Equation $38 \psi_{11}^{ \pm}=J_{1}\left(k_{c, 11}^{h} \rho\right) e^{\mp j \varphi}$. Since any pair of modes with $e^{-j \varphi}$ and $e^{j \varphi}$ azimuthal dependencies are orthogonal to each other and because of the phase matching condition in the graphene plane, any of these modes creates reflected and transmitted modes with the same azimuthal dependence. Therefore, the $\mathrm{H}_{11}^{-}$mode will excite the $\mathrm{H}_{1 n}^{-}$and $\mathrm{E}_{1 n}^{-}$modes, while the $\mathrm{H}_{11}^{+}$mode will excite the $\mathrm{H}_{1 n}^{+}$ans $\mathrm{E}_{1 n}^{+}$modes. Next, we focus on the $\mathrm{H}_{11}^{-}$mode. The analysis for the $\mathrm{H}_{11}^{+}$is completely similar.

The transverse reflected and transmitted fields in the graphene plane ( $z=0$ plane) read

$$
\begin{aligned}
\mathbf{E}_{T}^{-\mathrm{ref}} & =\sum_{n=1}^{\infty}\left(R_{1 n}^{h-} \mathbf{E}_{T, 1 n}^{h-}+R_{1 n}^{e-} \mathbf{E}_{T, 1 n}^{e-}\right), \\
\mathbf{E}_{T}^{-\operatorname{tran}} & =\sum_{n=1}^{\infty}\left(T_{1 n}^{h-} \mathbf{E}_{T, 1 n}^{h-}+T_{1 n}^{e-} \mathbf{E}_{T, 1 n}^{e-}\right),
\end{aligned}
$$

respectively. For the computation of the coefficients $R_{1 n}^{h-}, R_{1 n}^{e-}, T_{1 n}^{h-}$ and $T_{1 n}^{e-}$, the boundary conditions

$$
\begin{aligned}
\hat{\mathbf{z}} \times\left.\left(\mathbf{E}_{T}^{\text {tran }}-\mathbf{E}_{T}^{\mathrm{inc}}-\mathbf{E}_{T}^{\text {ref }}\right)\right|_{z=0} & =0, \\
\hat{\mathbf{z}} \times\left.\left(\mathbf{H}_{T}^{\text {tran }}-\mathbf{H}_{T}^{\mathrm{inc}}-\mathbf{H}_{T}^{\text {ref }}\right)\right|_{z=0} & =\left.\overline{\bar{\sigma}} \cdot \mathbf{E}_{T}^{\text {tran }}\right|_{z=0},
\end{aligned}
$$

should be used. The reflected and transmitted magnetic fields in the graphene plane are given by

$$
\begin{aligned}
\mathbf{H}_{T}^{- \text {ref }} & =-\sum_{n=1}^{\infty}\left(R_{1 n}^{h-} \frac{\hat{\mathbf{z}} \times \mathbf{E}_{T, 1 n}^{h-}}{Z_{1 n}^{h}}+R_{1 n}^{e-} \frac{\hat{\mathbf{z}} \times \mathbf{E}_{T, 1 n}^{e-}}{Z_{1 n}^{e}}\right), \\
\mathbf{E}_{T}^{-\operatorname{tran}} & =\sum_{n=1}^{\infty}\left(T_{1 n}^{h-} \frac{\hat{\mathbf{z}} \times \mathbf{E}_{T, 1 n}^{h-}}{Z_{1 n}^{h}}+T_{1 n}^{e-} \frac{\hat{\mathbf{z}} \times \mathbf{E}_{T, 1 n}^{e-}}{Z_{1 n}^{e}}\right),
\end{aligned}
$$

where $Z_{1 n}^{h}=\eta_{0}\left(k_{0} / \beta_{1 n}^{h}\right)$ and $Z_{1 n}^{e}=\eta_{0}\left(\beta_{1 n}^{e} / k_{0}\right)$ are the wave impedances of the $\mathrm{H}_{1 n}$ and $\mathrm{E}_{1 n}$ modes, respectively, and $\beta_{1 n}^{h}$ and $\beta_{1 n}^{e}$ are the corresponding propagation numbers. 
Inserting Equations 39 into 40a and using the orthogonality conditions (Collin, 1990)

$$
\begin{aligned}
& \int_{S} \mathbf{E}_{T, 1 n}^{h-} \cdot \mathbf{E}_{T, 1 m}^{h+}=\int_{S} \mathbf{E}_{T, 1 n}^{e-} \cdot \mathbf{E}_{T, 1 m}^{e+}=0, \quad n \neq m \\
& \int_{S} \mathbf{E}_{T, 1 n}^{h-} \cdot \mathbf{E}_{T, 1 m}^{e+}=\int_{S} \mathbf{E}_{T, 1 n}^{e-} \cdot \mathbf{E}_{T, 1 m}^{h+}=0,
\end{aligned}
$$

with $S$ the waveguide cross section, one obtains

$$
\begin{aligned}
R_{1 n}^{h-}+\delta_{1 n} & =T_{1 n}^{h-}, \\
R_{1 n}^{e-} & =T_{1 n}^{e-} .
\end{aligned}
$$

Similarly, introducing Equation 41 into $40 \mathrm{~b}$ yields

$$
\begin{array}{r}
-T_{1 n}^{h-}+\delta_{1 n}-R_{1 n}^{h-}=Z_{1 n}^{h} \sigma_{x x} T_{1 n}^{h-}+Z_{1 n}^{h} \sigma_{y x} \sum_{m=0}^{\infty}\left(J_{n m}^{h h} T_{1 m}^{h-}+J_{n m}^{h e} T_{1 m}^{e-}\right), \\
-T_{1 n}^{e-}-R_{1 n}^{e-}=Z_{1 n}^{e} \sigma_{x x} T_{1 n}^{e-}+Z_{1 n}^{e} \sigma_{y x} \sum_{m=0}^{\infty}\left(J_{n m}^{e h} T_{1 m}^{h-}+J_{n m}^{e e} T_{1 m}^{e-}\right),
\end{array}
$$

where

$$
\begin{aligned}
J_{n m}^{h h} & =\frac{\int_{S} \mathbf{E}_{1 n}^{h+} \cdot\left(\hat{\mathbf{z}} \times \mathbf{E}_{1 m}^{h-}\right) d S}{\int_{S} \mathbf{E}_{1 n}^{h+} \cdot \mathbf{E}_{1 n}^{h-} d S}, & J_{n m}^{h e} & =\frac{\int_{S} \mathbf{E}_{1 n}^{h+} \cdot\left(\hat{\mathbf{z}} \times \mathbf{E}_{1 m}^{e-}\right) d S}{\int_{S} \mathbf{E}_{1 n}^{h+} \cdot \mathbf{E}_{1 n}^{h-} d S}, \\
J_{n m}^{e h} & =\frac{\int_{S} \mathbf{E}_{1 n}^{e+} \cdot\left(\hat{\mathbf{z}} \times \mathbf{E}_{1 m}^{h-}\right) d S}{\int_{S} \mathbf{E}_{1 n}^{e+} \cdot \mathbf{E}_{1 n}^{e-} d S}, & J_{n m}^{e e} & =\frac{\int_{S} \mathbf{E}_{1 n}^{e+} \cdot\left(\hat{\mathbf{z}} \times \mathbf{E}_{1 m}^{e-}\right) d S}{\int_{S} \mathbf{E}_{1 n}^{e+} \cdot \mathbf{E}_{1 n}^{e-} d S} .
\end{aligned}
$$

In the derivation of Equation 45 the identity $\overline{\bar{J}}_{t} \cdot \mathbf{v}=\hat{\mathbf{z}} \times \mathbf{v}$, which holds for any vector $\mathbf{v}$, has been used. Omitting the proof, which can be found in (Sounas \& Caloz, 2011d), we directly give the final expressions of the $J$ coefficients of Equations 46

$$
\begin{array}{rlrl}
J_{n m}^{h h} & =-\frac{j 2}{\sqrt{\left[\left(x_{1 n}^{\prime}\right)^{2}-1\right]\left[\left(x_{1 m}^{\prime}\right)^{2}-1\right]}}, & & J_{n m}^{h e}=\frac{2\left(x_{1 n}^{\prime}\right)^{2}}{\left[\left(x_{1 n}^{\prime}\right)^{2}-x_{1 m}^{2}\right] \sqrt{\left(x_{1 n}^{\prime}\right)^{2}-1}}, \\
J_{n m}^{e h}=-\left(J_{m n}^{h e}\right)^{*}, & J_{n m}^{e e}=0,
\end{array}
$$

where $x_{1 n}$ and $x_{1 n}^{\prime}$ are the $n$th roots of $J_{1}(x)$ and $J_{1}^{\prime}(x)$, respectively. Solving Equations 44 with respect to $R_{1 n}^{h / e-}$ and introducing the result into Equations 45 we get

$$
\begin{gathered}
\left(2+Z_{1 n}^{h} \sigma_{x x}\right) T_{1 n}^{h-}+Z_{1 n}^{h} \sigma_{y x} \sum_{m=0}^{\infty}\left(J_{n m}^{h h} T_{1 m}^{h-}+J_{n m}^{h e} T_{1 m}^{e-}\right)=2 \delta_{1 n}, \\
\left(2+Z_{1 n}^{e} \sigma_{x x}\right) T_{1 n}^{e-}+Z_{1 n}^{e} \sigma_{y x} \sum_{m=0}^{\infty}\left(J_{n m}^{e h} T_{1 m}^{h-}+J_{n m}^{e e} T_{1 m}^{e-}\right)=0 .
\end{gathered}
$$

Equations 48 constitute a linear system of equations, the solution of which gives $T_{1 n}^{h / e-}$. One can calculate $T_{1 n}^{h / e+}$ in a similar manner. 
If the operation frequency is between the cutoff frequencies of the dominant mode and the first higher order mode, only the dominant mode exists far away from the graphene discontinuity, since all the higher order modes are evanescent. Then, similar to the case of plane wave incidence on graphene in free space, the rotation angle of the transmitted mode is

$$
\theta=\frac{\varphi^{-}-\varphi^{+}}{2}
$$

where here $\varphi^{ \pm}=\arg \left\{T_{11}^{h \pm}\right\}$.

An approximate closed-form expression for $\theta$ can be obtained by considering only the dominant mode terms in Equations 48. The solution of Equations 48 reads then

$$
T_{11}^{h \pm}=\frac{2}{\left(2+Z_{11}^{h} \sigma_{x x}\right) \pm j \alpha Z_{11}^{h} \sigma_{y x}},
$$

with $\alpha=2 /\left[\left(x_{11}^{\prime}\right)^{2}-1\right]$. Subsequently, the rotation angle is found as

$$
\theta=\tan ^{-1}\left(\frac{\alpha Z_{11}^{h} \sigma_{y x}}{2+Z_{11}^{h} \sigma_{x x}}\right) .
$$

The frequency dependence of the rotation angle and the transmission amplitude, computed exactly via Equation 49 and approximately via Equation 51, are presented in Fig. 15 for a waveguide with $a=8 \mathrm{~cm}$ and a graphene sheet with $\tau=1.84 \times 10^{-13} \mathrm{~s}, \mu_{c}=0.3 \mathrm{eV}$ and $B_{0}=$ $1 \mathrm{~T}$. The rotation angle and the transmission amplitude for normal incidence on graphene in free space, computed through Equation 36, are also plotted for comparison. Very good agreement between the exact (Equation 49) and the approximate (Equation 51) formulas is observed.

Fig. 15 shows that $\theta$ decreases as frequency increases. This may be understood from the frequency behavior of $Z_{11}^{h}$, which tends to $\infty$ at the cutoff of the $\mathrm{H}_{11}$ mode and increases towards $\eta_{0}$ as frequency increases. At the $\mathrm{H}_{11}$ mode cutoff frequency, $\theta$ takes its maximum value

$$
\theta=\tan ^{-1}\left(\alpha \frac{\sigma_{y x}}{\sigma_{x x}}\right)
$$

However, the transmission amplitude close to cutoff is very small. On the other hand, one would expect that at $\omega \rightarrow \infty$, when the $\mathrm{H}_{11}$ mode propagates almost parallel to the waveguide axis and $Z_{11}^{h} \rightarrow \eta_{0}, \theta$ would tend to its free space value (Equation 36), whereas, as seen from Equation 51, it tends to a smaller value ${ }^{5}$. A physical explanation can be given as follows. As the $\mathrm{H}_{11}$ wave impinges on graphene, it produces a transverse current component, which is responsible for the polarization rotation. However, this current does not perfectly match the transverse pattern of the mode and therefore only partially contributes to the transmitted field amplitude, thus providing a smaller rotation angle than in free space. This mismatch is also responsible for the excitation of the higher order modes in the waveguide environment.

\footnotetext{
${ }^{5} \alpha<1$.
} 


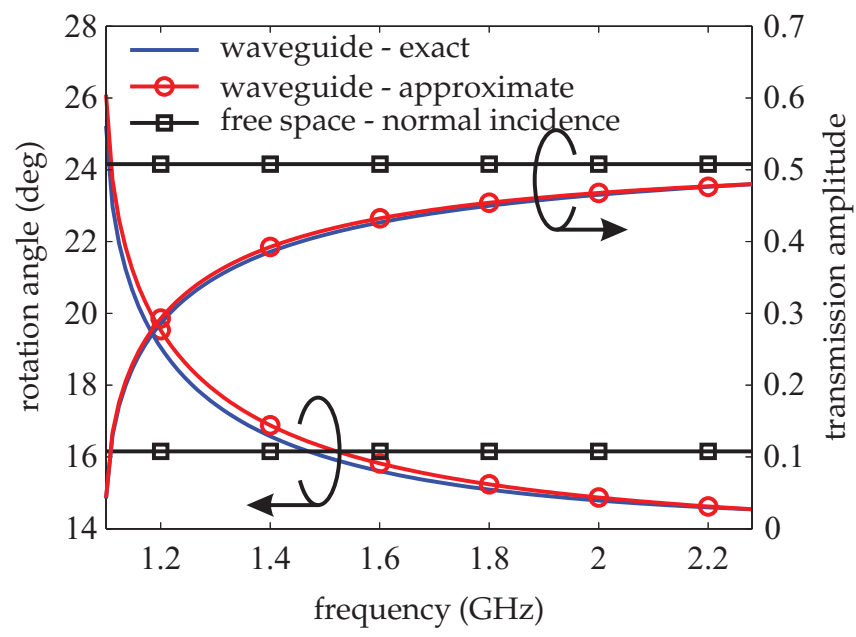

Fig. 15. Frequency dependence of the rotation angle and the transmission amplitude for a waveguide, computed exactly by Equations 48 and 49 and approximately by Equations 50 and 51 , for a waveguide with $a=8 \mathrm{~cm}$ and a graphene sheet with $\tau=1.84 \times 10^{-13} \mathrm{~s}$, $\mu_{c}=0.3 \mathrm{eV}, B_{0}=1 \mathrm{~T}$ and $\mathrm{T}=300 \mathrm{~K}$.

\subsection{Spatial isolator}

As a second application of the gyrotropic properties of graphene, we propose the spatial isolator of Fig. 16 (Sounas \& Caloz, 2011c). It consists of a magnetically biased graphene sheet in the middle of two wire grids, which are rotated by an angle of $45^{\circ}$ with respect to each other. The structure allows propagation of a $y$-polarized wave from medium 1 towards medium 2, while it blocks any wave propagating from medium 2 towards medium 1.

The operation principle of the structure is the same as of the ferrite-based non-reciprocal spatial isolator presented in (Parsa et al., 2010). A $y$-polarized wave propagating along the $-z$ direction passes through the wire grid at $z=d_{1}$, it is then rotated by $45^{\circ}$ in the LH direction with respect to the $z$-axis due to graphene and it eventually passes through the wire grid at $z=d_{2}$. On the other hand, a wave propagating along the $+z$ direction is directly blocked by the grid at $z=d_{2}$ if it is polarized parallel to the wires of this grid. If it is perpendicularly polarized to the wires of this grid, it passes through this grid, it is then rotated by $45^{\circ}$ in the LH direction as it passes through graphene and it is eventually blocked by the grid at $z=d_{1}$.

The structure can be analyzed by using the transmission line model of Fig. 17. In this model graphene and the wire grids are represented by shunt dyadic admittances and the space between graphene and the grids by transmission line sections with wave impedance $\eta_{0}$ and propagation number $k_{0}$. The shunt admittance which models the graphene sheet is simply the tensorial conductivity of graphene (Equation 13). The shunt admittance which models the grid at $z=d_{1}$ is

$$
\overline{\bar{Y}}_{1}=Y_{g \|} \hat{\mathbf{x}} \hat{\mathbf{x}}+Y_{g \perp} \hat{\mathbf{y}} \hat{\mathbf{y}}
$$

where

$$
Y_{g \|}=j \eta_{0}^{-1} \frac{\lambda}{d} \frac{1}{\ln \left[\sin \left(\frac{\pi w}{2 d}\right)\right]}
$$




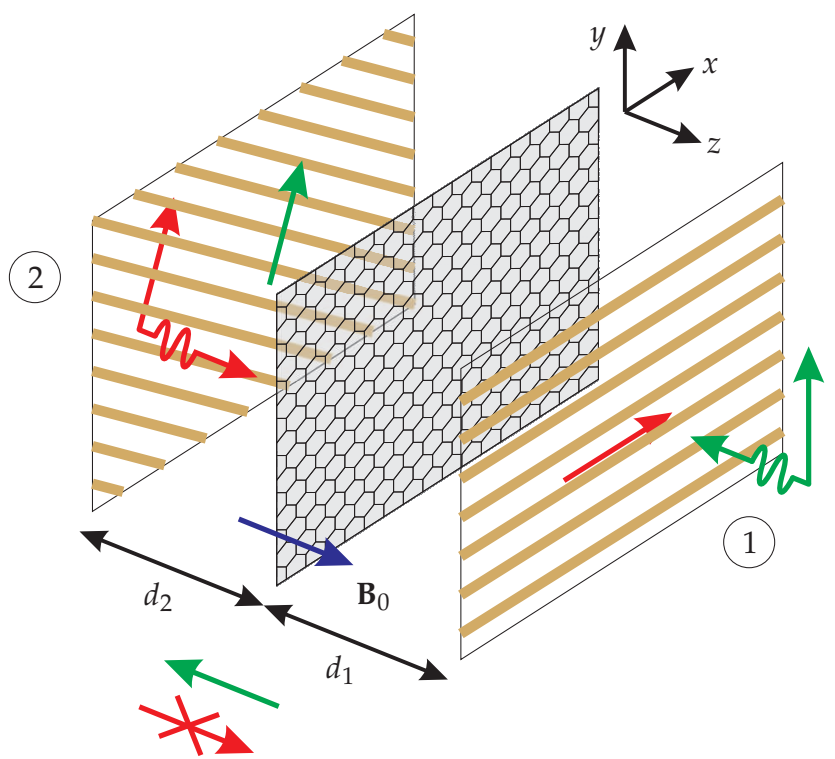

Fig. 16. Graphene-based non-reciprocal spatial isolator, consisting of a graphene sheet and two wire grids, one with wires parallel to the $x$-axis and the other with wires tilted by $-45^{\circ}$ with respect to the $x$-axis. A $y$-polarized wave propagating along the $-z$-direction passes through the isolator, while any wave propagating along the $z$-direction does not.

and

$$
Y_{g \perp}=-j \eta_{0}^{-1} \frac{4 d}{\lambda} \ln \left\{\sin \left[\frac{\pi(d-w)}{2 d}\right]\right\}
$$

are the parallel and the perpendicular to the wires admittances of the grid, respectively (Tretyakov, 2003). In Equation $54 w$ is the wire width and $d$ is the periodicity of the grid. The shunt admittance of the grid at $z=d_{2}$ is

$$
\bar{Y}_{2}=Y_{g \|} \hat{\mathbf{t}} \hat{\mathbf{t}}+Y_{g \perp}(\hat{\mathbf{z}} \times \hat{\mathbf{t}})(\hat{\mathbf{z}} \times \hat{\mathbf{t}}),
$$

where $\hat{\mathbf{t}}=(\hat{\mathbf{x}}+\hat{\mathbf{y}}) / \sqrt{2}$ is the unit vector parallel to the wires.

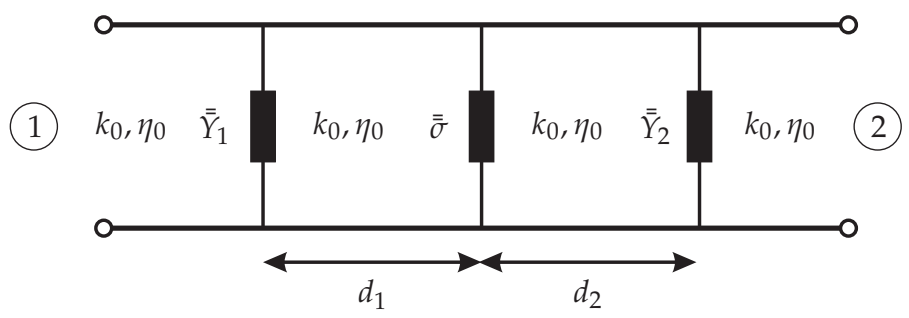

Fig. 17. Transmission line model of the proposed graphene-based non-reciprocal spatial isolator. 
The $A B C D$ matrix of the structure, through which the transmission coefficients will be computed, is

$$
\begin{aligned}
{\left[\begin{array}{cc}
\overline{\bar{A}} & \overline{\bar{B}} \\
\overline{\bar{C}} & \overline{\bar{D}}
\end{array}\right]=} & {\left[\begin{array}{cc}
\overline{\bar{I}} & \overline{\overline{0}} \\
\overline{\bar{Y}} & \overline{\bar{I}}
\end{array}\right] \cdot\left[\begin{array}{cc}
\cos \left(k_{0} d_{1}\right) & j \eta_{0} \sin \left(k_{0} d_{1}\right) \\
j \eta_{0}^{-1} \sin \left(k_{0} d_{1}\right) & \cos \left(k_{0} d_{1}\right)
\end{array}\right] \cdot\left[\begin{array}{cc}
\overline{\bar{I}} & \overline{0} \\
\overline{\bar{\sigma}} & \overline{\bar{I}}
\end{array}\right] } \\
& \cdot\left[\begin{array}{cc}
\cos \left(k_{0} d_{2}\right) & j \eta_{0} \sin \left(k_{0} d_{2}\right) \\
j \eta_{0}^{-1} \sin \left(k_{0} d_{2}\right) & \cos \left(k_{0} d_{2}\right)
\end{array}\right] \cdot\left[\begin{array}{cc}
\overline{\bar{I}} & \overline{\overline{0}} \\
\overline{\bar{Y}} & \overline{\bar{I}}
\end{array}\right] \cdot
\end{aligned}
$$

The S-matrix is then

$$
[\overline{\bar{S}}]=\left[\begin{array}{cr}
\overline{\bar{I}} & -\left(\overline{\bar{A}}+\eta_{0} \overline{\bar{B}}\right) \\
-\eta_{0} & \overline{\bar{I}}-\left(\overline{\bar{C}}+\eta_{0} \overline{\bar{D}}\right)
\end{array}\right]^{-1} \cdot\left[\begin{array}{cc}
-\overline{\bar{I}} & \overline{\bar{A}}-\eta_{0} \overline{\bar{B}} \\
-\eta_{0} \overline{\bar{I}} & \overline{\bar{C}}-\eta_{0} \overline{\bar{D}}
\end{array}\right]
$$

and the isolation may be subsequently computed as

$$
I=20 \log _{10}\left|\bar{S}_{21} \cdot \hat{\mathbf{y}}\right|-20 \log _{10}\left|\overline{\bar{S}}_{12} \cdot \frac{\hat{\mathbf{x}}+\hat{\mathbf{y}}}{\sqrt{2}}\right| .
$$

Fig. 18 presents isolation in $\mathrm{dB}$ (color plot), the transmission loss from medium 1 to medium 2 in $\mathrm{dB}$ (continuous lines contour plot) and the rotation angle of graphene in free space (dashed lines contour plot) versus $\mu_{c}$ and $B_{0}$ for $\tau=1.84 \times 10^{-13} \mathrm{~s}$. The results are derived for $d_{1}=d_{1}=\lambda / 4$, which has been found to be the condition of the highest isolation. As an example of how to read Fig. 18, the point indicated by the circle corresponds to $B_{0}=0.61 \mathrm{~T}$, $\mu_{c}=0.11 \mathrm{eV}, I=20 \mathrm{~dB}$, a $3 \mathrm{~dB}$ transmission loss from medium 1 towards medium 2 and $9^{\circ}$ of rotation angle for graphene in free space. One observes that the isolation can be so high as $20 \mathrm{~dB}$ even when the rotation angle of graphene in free space is so low as $10^{\circ}$. This contrasts with the ferrite-based isolator, where a $45^{\circ}$ rotation angle is always required. This surprising behavior is attributed to the semi-transparent nature of graphene which forces the wave to follow a complex path between the grids, passing several times through graphene. The condition for which this complex path leads to constructive interference for propagation along the $+z$ direction and destructive interference for propagation along the $-z$ direction is $d_{1}=d_{2}=\lambda / 4$. The main reason for the relatively high loss $(3 \mathrm{~dB})$ for transmission along the $+z$ direction is the graphene loss, which is increased by the large effective wave path in the space between the grids. This loss can be reduced by using graphene with higher mobility (lower scattering) and properly adjusting the structure parameters.

One of the most significant advantages of the proposed isolator is the control of the isolation direction via the chemical potential, exploiting the ambipolar properties of graphene. In particular, by inverting the chemical potential, the direction of Faraday rotation provided by graphene also inverts, which in turn inverts the isolation direction. Remember that the chemical potential can be easily controlled via an electrostatic voltage applied between graphene and an electrode parallel to graphene (Fig. 2). In such a way it could be possible to fabricate a non-reciprocal antenna radome, which dynamically converts an antenna from transmitting to receiving and vice versa through a proper control signal.

\section{Enabling of graphene gyrotropy with metamaterials}

As already pointed out, the structures presented in Section 5 are essentially "proofs of concept" and do not follow an optimal design. For example, a practical circular waveguide 


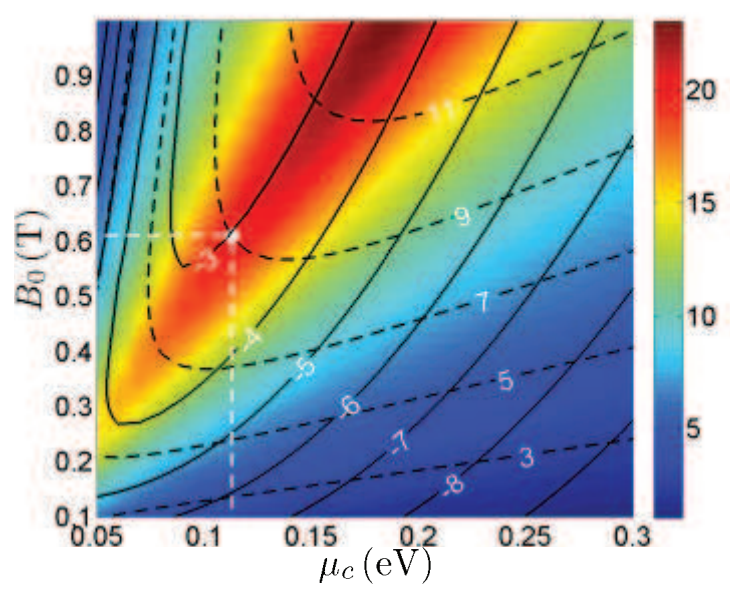

Fig. 18. Color plot: isolation in $\mathrm{dB}$ achieved by the proposed graphene-based non-reciprocal spatial isolator versus $\mu_{c}$ and $B_{0}$ for $\tau=1.84 \times 10^{-13}$ s. Solid lines contour plot: power transmission coefficient in $\mathrm{dB}$ for the wave allowed to pass through the isolator. Dashed lines contour plot: polarization rotation angle of the graphene sheet in free space.

Faraday rotator, which is a necessary component in a waveguide isolator or a gyrator, requires a rotation angle of at least $90^{\circ}$. Although the rotation angle provided by a single graphene sheet is huge considering its one-atom thickness, it is quite far from the value required in a waveguide isolator. In order to achieve a rotation angle so high as $90^{\circ}$ it is necessary to stack several graphene sheets. The material and the spacing between the sheets as well as the rotation angle provided by each sheet must be carefully chosen so that the transmission through such a structure be maximum. In such a way, a microscale metamaterial, similar to this illustrated in Fig. 19(a), can be conceived. Such a metamaterial provides a gradual rotation of the wave polarization, thus mimicking a ferrite medium with, however, a much larger

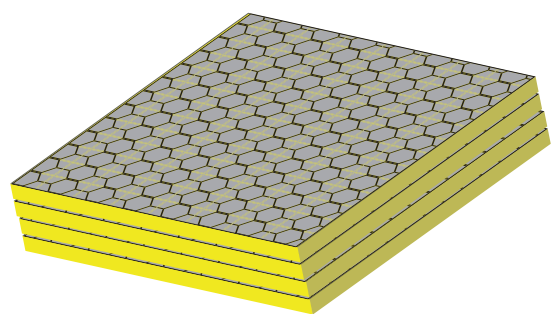

(a)

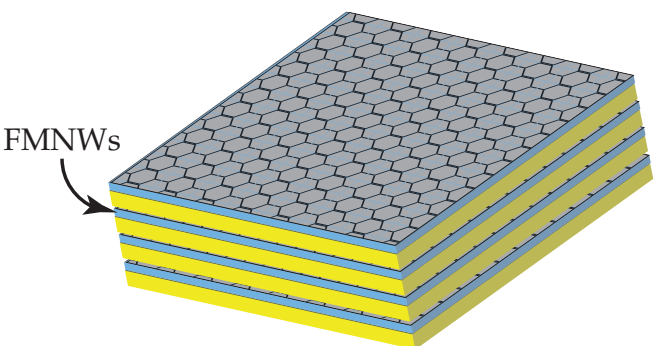

(b)

Fig. 19. Graphene-based multiscale metamaterials, which exhibit a ferrite-type response, however, with a higher rotation power than ferrites. (a) Micro-scale metamaterial consisting of a period stack of graphene sheets and dielectric layers. (b) Multiscale metamaterial consisting of a periodic stack of graphene sheets, ferromagnetic nanowires (FMNW) membranes and dielectric layers. FMNW metamaterial membranes are used for biasing graphene. 
Verdet constant (rotation per unit thickness and unit magnetic field). Such an artificial material could be also used in the spatial isolator to reduce significantly its size. In a next nanoscale level, ferromagnetic nanowires membranes (Carignan et al., 2011), which are transparent to normally incident waves, could be introduced between the successive graphene layers, as illustrated in Fig. 19(b) to provide the necessary static magnetic field, making the material self-biased. Finally, boron nitride (BN) could be selected as the separating dielectric between the graphene sheets, since as it has been recently shown, graphene sandwiched between BN layers exhibits extremely high mobility, sometimes approaching that of suspended graphene (Mayorov et al., 2011). It is therefore clear that by exploiting metamaterials concept in the micro, nano and atomic scale simultaneously (Caloz et al., n.d.), novel material with unprecedented properties can be created.

\section{Conclusions}

The gyrotropic properties of magnetically biased graphene have been analyzed. Graphene exhibits strong non-reciprocity and extremely broadband Faraday rotation at microwave frequencies, below the cyclotron resonance. Two degrees of freedom may be used to control the amount and the direction of rotation, namely the chemical potential (via an applied static voltage) and the static magnetic field. This allows full tuning of Faraday rotation via the chemical potential, while keeping the magnetic field constant, which is usually provided by permanent magnets and is thus difficult to control. Two applications of graphene gyrotropy have been proposed: a circular waveguide Faraday rotator and a spatial isolator. A practical realization of such devices with unprecedented characteristics, such as super compactness, is possible through the multi-scale (micro, nano and atomic scales) metamaterial concept.

\section{References}

Abramowitz, M. \& Stegun, I. A. (1964). Handbook of mathematical functions with formulas, graphs, and mathematical tables, Vol. 55, Courier Dover publications.

Balanis, C. A. (2005). Antenna theory: analysis and design, John Wiley \& Sons.

Caloz, C., Carignan, L.-P., Ménard, D. \& Yelon, A. (n.d.). The concept of multi-scale metamaterials.

Caloz, C. \& Itoh, T. (2006). Electromagnetic Metamaterials: Transmission Line Theory, and Microwave Applications: The Engineering Approach, John Wiley \& Sons.

Carignan, L.-P., Yelon, A., Ménard, D. \& Caloz, C. (2011). Ferromagnetic nanowire metamaterials: theory and applications, IEEE Trans. Microwave Theory Tech. 59(10): 2568-2586.

Collin, R. E. (1990). Field theory of guided waves, IEEE Press.

Crassee, I., Levallois, J., Walter, A. L., Ostler, M., Bostwick, A., Rotenberg, E., Seyller, T., van der Marel, D. \& Kuzmenko, A. B. (2011). Giant faraday rotation in single- and multilayer graphene, Nature Phys. 7: 48-51.

Dragoman, M. \& Dragoman, D. (2009). Nanoelectronics: principles and devices, Artech House.

Geim, A. K. \& Novoselov, K. S. (2007). The rise of graphene, Nature Materials 6: 183-191.

Gusynin, V. P., Sharapov, S. G. \& Carbotte, J. P. (2009). On the universal ac optical background in graphene, New J. Phys. 11: 095013.

Hanson, G. W. (2007). Fundamentals of Nanoelectronics, Prentice Hall.

Koppens, F. H. L., Chang, D. E. \& García de Abajo, F. J. (2011). Graphene plasmonics: A platform for strong light-matter interactions, Nano Letters 11(8): 3370-3377. 
Kubo, R. (1957). Statistical-mechanical theory of irreversible processes. I. general theory and simple applications to magnetic and conduction problems, J. Phys. Soc. Japan 12(6): 570-586.

Lax, B. \& Button, K. J. (1962). Microwave Ferrites and Ferrimagnetics, McGraw-Hill.

Lin, Y.-M., Jenkins, K. A., Valdes-Garcia, A., Small, J. P., Farmer, D. B. \& Avouris, P. (2009). Operation of graphene transistors at gigahertz frequencies, Nano Lett. 9: 422-426.

Lindell, I. V. (1996). Methods for electromagnetic field analysis, IEEE Press.

Marqués, R., Martín, F. \& Sorolla, M. (2008). Metamaterials with Negative Parameters: Theory, Design and Microwave Applications, John Wiley \& Sons.

Mayorov, A. S., Gorbachev, R. V., Morozov, S. V., Britnell, L., Jalil, R., Ponomarenko, L. A., Blake, P., Novoselov, K. S., Watanabe, K., Taniguchi, T. \& Geim, A. K. (2011). Micrometer-scale ballistic transport in encapsulated graphene at room temperature, Nano Lett. 11(6): 2396-2399.

Mikhailov, S. A. \& Ziegler, K. (2007). New electromagnetic mode in graphene, Phys. Rev. Lett. 99: 016803.

Mishchenko, E. G., Shytov, A. V. \& Silvestrov, P. G. (2010). Guided plasmons in graphene $p-n$ junctions, Phys. Rev. Lett. 104: 156806.

Neto, A. H. C., Guinea, F., Peres, N. M. R., Novoselov, K. S. \& Geim, A. K. (2009). The electronic properties of graphene, Rev. Mod. Phys. 81: 109-162.

Novoselov, K. S., Geim, A. K., Morozov, S. V., Jiang, D., Zhang, Y., Dubonos, S. V., Grigorieva, I. V. \& Firsov, A. A. (2004). Electric field effect in atomically thin carbon films, Science 306: 666-669.

Parsa, A., Kodera, T. \& Caloz, C. (2010). Ferrite based non-reciprocal radome, generalized scattering matrix analysis and experimental demonstration, IEEE Trans. Antennas Propag. . in press.

Sounas, D. L. \& Caloz, C. (2011a). Edge surface modes in magnetically-biased chemically-doped graphene strips, App. Plys. Lett. . under review.

Sounas, D. L. \& Caloz, C. (2011b). Electromagnetic non-reciprocity and gyrotropy of graphene, Appl. Phys. Lett. 98: 021911.

Sounas, D. L. \& Caloz, C. (2011c). Graphene-based non-reciprocal spatial isolator, Proc. IEEE International Symposium on Antennas and Propagation, Spokane, WA, (APS 2011).

Sounas, D. L. \& Caloz, C. (2011d). Gyrotropy and non-reciprocity of graphene for microwave applications, IEEE Trans. Microw. Theory Tech. . under review.

Tretyakov, S. (2003). Analytical modelling in applied electromagnetics, Artech House, Inc.

Vakil, A. \& Engheta, N. (2011). Transformation optics using Graphene, Science 332(6035): 1291-1294.

Wang, H., Nezich, D., Kong, J. \& Palacios, T. (2009). Graphene frequency multipliers, IEEE Electron Device Lett. 30: 547-549. 


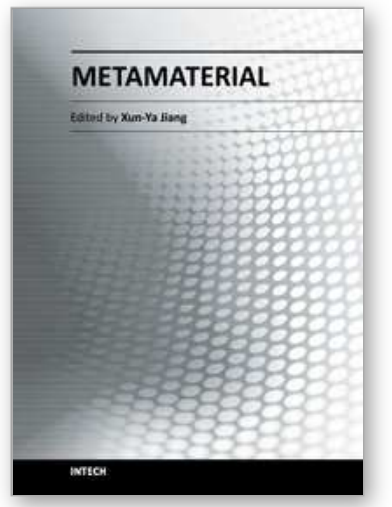

\author{
Metamaterial \\ Edited by Dr. Xun-Ya Jiang
}

ISBN 978-953-51-0591-6

Hard cover, 620 pages

Publisher InTech

Published online 16, May, 2012

Published in print edition May, 2012

In-depth analysis of the theory, properties and description of the most potential technological applications of metamaterials for the realization of novel devices such as subwavelength lenses, invisibility cloaks, dipole and reflector antennas, high frequency telecommunications, new designs of bandpass filters, absorbers and concentrators of EM waves etc. In order to create a new devices it is necessary to know the main electrodynamical characteristics of metamaterial structures on the basis of which the device is supposed to be created. The electromagnetic wave scattering surfaces built with metamaterials are primarily based on the ability of metamaterials to control the surrounded electromagnetic fields by varying their permeability and permittivity characteristics. The book covers some solutions for microwave wavelength scales as well as exploitation of nanoscale EM wavelength such as visible specter using recent advances of nanotechnology, for instance in the field of nanowires, nanopolymers, carbon nanotubes and graphene. Metamaterial is suitable for scholars from extremely large scientific domain and therefore given to engineers, scientists, graduates and other interested professionals from photonics to nanoscience and from material science to antenna engineering as a comprehensive reference on this artificial materials of tomorrow.

\title{
How to reference
}

In order to correctly reference this scholarly work, feel free to copy and paste the following:

Dimitrios L. Sounas and Christophe Caloz (2012). Novel Electromagnetic Phenomena in Graphene and Subsequent Microwave Devices Enabled by Multi-Scale Metamaterials, Metamaterial, Dr. Xun-Ya Jiang (Ed.), ISBN: 978-953-51-0591-6, InTech, Available from: http://www.intechopen.com/books/metamaterial/novelelectromagnetic-phenomena-in-graphene-and-subsequent-microwave-devices-enabled-by-multi-scale-

\section{INTECH}

open science | open minds

\section{InTech Europe}

University Campus STeP Ri

Slavka Krautzeka 83/A

51000 Rijeka, Croatia

Phone: +385 (51) 770447

Fax: +385 (51) 686166

www.intechopen.com
InTech China

Unit 405, Office Block, Hotel Equatorial Shanghai

No.65, Yan An Road (West), Shanghai, 200040, China

中国上海市延安西路65号上海国际贵都大饭店办公楼405单元

Phone: +86-21-62489820

Fax: +86-21-62489821 
(C) 2012 The Author(s). Licensee IntechOpen. This is an open access article distributed under the terms of the Creative Commons Attribution 3.0 License, which permits unrestricted use, distribution, and reproduction in any medium, provided the original work is properly cited. 